PAEDIATRIC LUNG DISEASE

\title{
A new method for objective identification and measurement of airway lumen in paediatric flexible videobronchoscopy
}

\author{
I B Masters, M M Eastburn, R Wootton, R S Ware, P W Francis, P V Zimmerman, A B Chang
}

Thorax 2005;60:652-658. doi: 10.1136/thx.2004.034421

See end of article for authors' affiliations

\section{Correspondence to:}

Dr I B Masters, Department of Respiratory Medicine, Royal Children's Hospital, Herston 4029, Brisbane, Australia; brent_masters@ health.qld.gov.au

Received 6 September 2004

Accepted 17 March 2005
Background: Accurate measurements of airway and lesion dimensions are important to the developmental progress of paediatric bronchoscopy. The malacia disorders are an important cause of respiratory morbidity in children, but no methods are currently available to measure these lesions or the airway lumen accurately. A new measurement technique is described here.

Methods: The magnification power of a paediatric videobronchoscope was defined and a simple and user friendly computer based program (Image J) was used to develop an objective technique (colour histogram mode technique, CHMT) for measurement of the airway lumen.

Results: In vivo intra-observer and inter-observer repeatability coefficients for repeated area measurements from 28 images using the Bland-Altman method were $0.9 \mathrm{~mm}^{2}$ and $1.6 \mathrm{~mm}^{2}$, respectively. The average intraclass correlation coefficient for repeated measurements of area was 0.93 . In vitro validation measurements using a $2 \mathrm{~mm}$ diameter tube resolved radii measurements to within $0.1 \mathrm{~mm}$ (coefficient of variability $8 \%$ ). An "acceptable result" was defined in $92 \%$ of 734 images completed with the CHMT alone and $8 \%$ with its modification. The success rate for two of three images being within $10 \%$ of each other's area was $100 \%$. Measurements of cricoid cross sectional areas from 116 patients compared with expected airway areas for age derived from endotracheal tube sizes were comparable.

Conclusions: The CHMT method of identifying and measuring airway dimensions is objective, accurate, and versatile and, as such, is important to the future development of flexible videobronchoscopy.
$\mathrm{T}$ he capacity for accurate identification of airway lumen, its dimensions and objective measurement is an important but yet to be realised development for a paediatric bronchoscopist. Indeed, as the role of flexible videobronchoscopy (FVB) continues to expand in clinical and research work, incorporation of accurate real time measurements of distance, airway cross sectional area, and size of lesions or their impact on cross sectional area would be a major advantage, particularly when assessing hypoplastic airways, malacia disorders, and stenoses that may involve both the lower and upper airways. ${ }^{1-5}$ In addition, it would enable an understanding of the effects of the dynamics of lung mechanics and lung volume variations. ${ }^{267}$

A variety of techniques from simple square counting to sophisticated computing techniques and ultrasound have been developed and modified to measure the cross sectional area of airways or specific sites within the airway, but none have been incorporated in the routine workings of paediatric bronchoscopic instruments. ${ }^{6-15}$ This situation is currently influenced by limiting factors in the engineering of bronchoscopes and image processing, which include the inability to accurately define the position and orientation of the bronchoscope lens in space; the effects of distance on magnification; the effects of variable magnification across the field of view; and the effects of geometric, radial or curvilinear, and anamorphic distortion. ${ }^{16}{ }^{17}$

For image processing of an airway or lesion to be carried out, the computing techniques must identify the outline of the airway rapidly and accurately. Once these data are obtained, the magnification power of the bronchoscope and the operating distance from the object are prerequisites to calculation of actual size. Currently, estimates of distance can be made but cross sectional area and diameter measurement and apparent loss of area are either descriptive and subjective approximations or relative changes in area across the respiratory cycle. These have generally been performed with visual inspection methods where intra-observer and interobserver variations are likely to be high or, in some cases, not defined. ${ }^{2}{ }^{8}$ These impressions of area or size of a lesion may be reinforced by passage of the bronchoscope or an endotracheal tube into and through lesions where this is physically possible. ${ }^{18}$ The major problem with this impression is the lack of reference data and a reference point from which to make objective comparisons. Even though there are data on relative proportions of the components of the airway, there are no longitudinal or cross sectional study data for children but, more importantly, there are none that take into account respiratory cycle timing. ${ }^{19-22}$ Currently, clinical decisions are made from relative and subjective approximations used in conjunction with the symptoms and signs of the patient. ${ }^{23}$

This paper describes a new and user friendly method to define and measure the airway lumen using a paediatric FVB and readily accessible and inexpensive computer software. We describe the intra-observer and inter-observer reliability of this technique, its in vitro validation, and its in vivo application, and compare airway size measurements using this technique with measurements using different methodologies.

\section{METHODS}

Real time FVB images captured by a digital video camera (Sony Mini DV Digital Handycam, Sony Corporation, Tokyo, Japan) were downloaded digitally to a laptop computer (MAC Power Book G4, Apple Inc, Cupertino, CA, USA) using an image capturing program (BTV Pro Carbon, Ben Bird) and an image processing program (Image J, Wayne Rasband, National Institute of Health, USA) that enabled airway lumen identification and cross sectional area measurement. The calibration and measurement of the bronchoscopic

Abbreviations: CHMT, colour histogram mode technique; FVB, flexible videobronchoscopy 
magnification power, reliability testing, validation of the technique, and in vivo testing are described below.

\section{Magnification and calibration of videobronchoscope} Magnification power of the bronchoscope (Olympus BF type 3C160) was derived by comparing the image size of an object to actual size of the object at specific distances from the object. The details of the derivation of magnification factors for the bronchoscope have been published and contain tables of magnification factors for a range of working distances: at a distance of $10 \mathrm{~mm}$ from the object the magnification was $\times 9.55 .^{24}$

\section{Identification and calculation of cross sectional area of airways}

The identification of the airway lumen and cross sectional area for measurement were derived in the following way by using the "Tools" function, the Image "Adjust Colour Balance" function, and the Analysis "Region of Interest" function of the Image $J$ software. The image of the airway captured by the BTV program was processed in 24-bit RGB colour and the histogram of the colour band (pixel number and intensity) as obtained by the image $J$ program was divided at the mode point by the maximal and minimal brightness moveable line-that is, placing the line at the same point or in the vertical plane through the mode point (highest point) of the image's colour histogram. This resulted in red, yellow, white and black colours, the black area being formed in the darkest part of the field of view-the luminal orifice of the selected airways. The Wand (tracing) tool was then selected and applied to the black area. This resulted in the outline of the black area or airway lumen being defined. The cross sectional area was calculated with the region of interest (ROI) and selection measurement functions. The real size of the image was then calculated by correcting for the magnification factor at that particular distance. This technique was referred to as the "colour histogram mode technique" (CHMT). Figures 1-3 show examples of the images and the defined airway lumen for measurement from a variety of sites within the airway that were obtained by the methodology described above. These three frames of each image also demonstrate the capacity to identify objectively the lumen from various sites within the paediatric airway. Frame 1 displays the actual airway image; frame 2 displays the area identified and defined for measurement and the superimposed colour histogram; and frame 3 displays the outline of the area measured in each sequence superimposed on each of the original airway images.

The mechanism by which the measurements are made with the Image $\mathrm{J}$ colour balance function is as follows. The original image was captured as a 24-bit RGB image from which a histogram of the colours is formed with 256 brightness bins from the eight colours (red, green, blue, cyan, magenta, yellow, black and white). The bin with the greatest pixel count is the mode point. The mode point and surrounding brightness bins on all of the images are a result of the tissues reflecting the light that surround the airway orifice. Once the mode point is defined and the minimum and maximum brightness levels are set to the mode point, the original image will only display the eight palette colours, so anything that is darker than the mode point (such as the airway of interest) will be shaded black and all other parts will be shaded one of the remaining seven colours (predominantly red, yellow and white). This then allows the area of interest (airway lumen) to be clearly marked and measured with a pixel count of a set scale through the Tools function and ROI manager functions of the Image $\mathrm{J}$ Program.

\section{Validation and reliability testing}

Validation testing

Validation experiments were carried out using a drilled opaque white plastic tube of $2 \mathrm{~mm}$ internal diameter and $20 \mathrm{~mm}$ long. Caliper measurement of the internal diameter
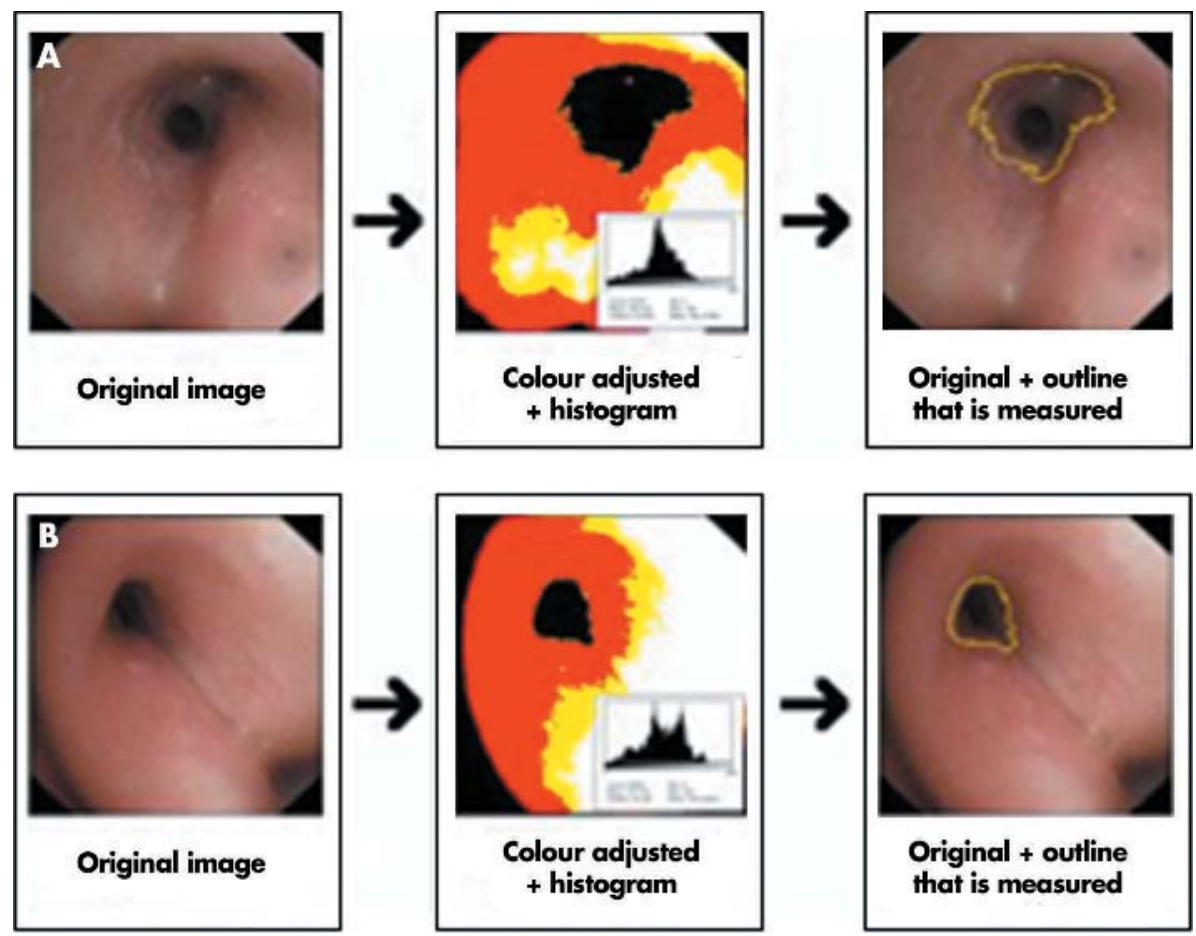

Figure 1A,B Transitions in the image created by the process of dividing the colour scales of the images around the mode point of the histogram as shown in the insert. The final image shows the area to be measured superimposed on the actual image. This visually displays the representative accuracy of the measurement. The images shown are the right main stem bronchus and the left main stem bronchus which is stenotic. 

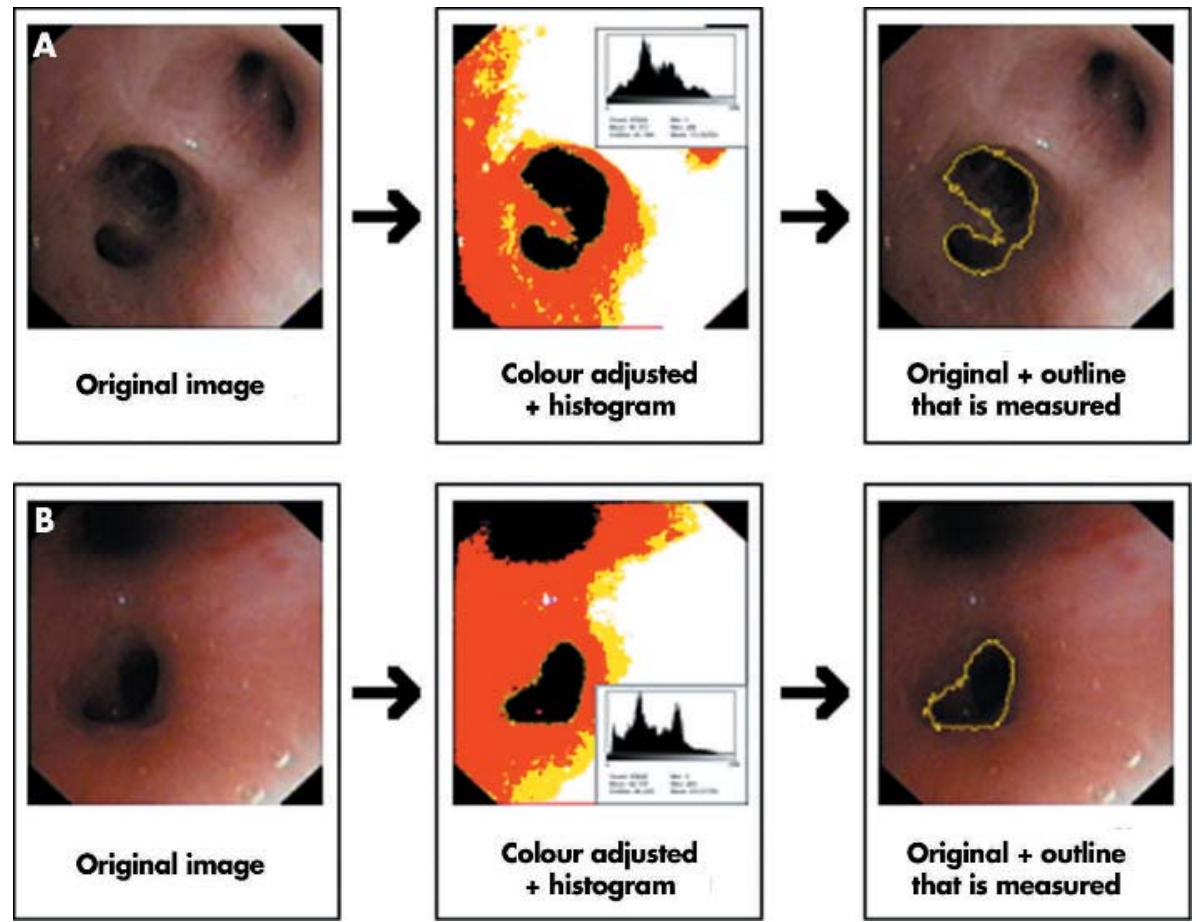

Figure 2A,B Transitions in the image created by the colour histogram. (A) The bronchus outlined is the lower end of the bronchus intermedius with the middle lobe at the $2 o^{\prime}$ clock position. (B) The outline of the left lower lobe bronchus with the left upper lobe orifice at the 12 o'clock position.

varied between $2.02 \mathrm{~mm}$ and $2.05 \mathrm{~mm}$. These tubes were placed at the end of an airway model such that distances from the tip of the bronchoscope to the object and the distances moved at the position of entry could be compared. These distances were measured with an electronic digital caliper to the nearest $0.002 \mathrm{~mm}$. The bronchoscope was hand held at the entry point and a peg was placed at the zero point (bronchoscope tip touching the object); the bronchoscope was then withdrawn prescribed distances of 30, 20, 15 and $10 \mathrm{~mm}$ respectively from the object with the image positioned as close as possible to the centre of the screen. In all cases the longitudinal movement of the tip of the bronchoscope from the object was reflected by exactly the same measurement between the marker peg and the ultimate
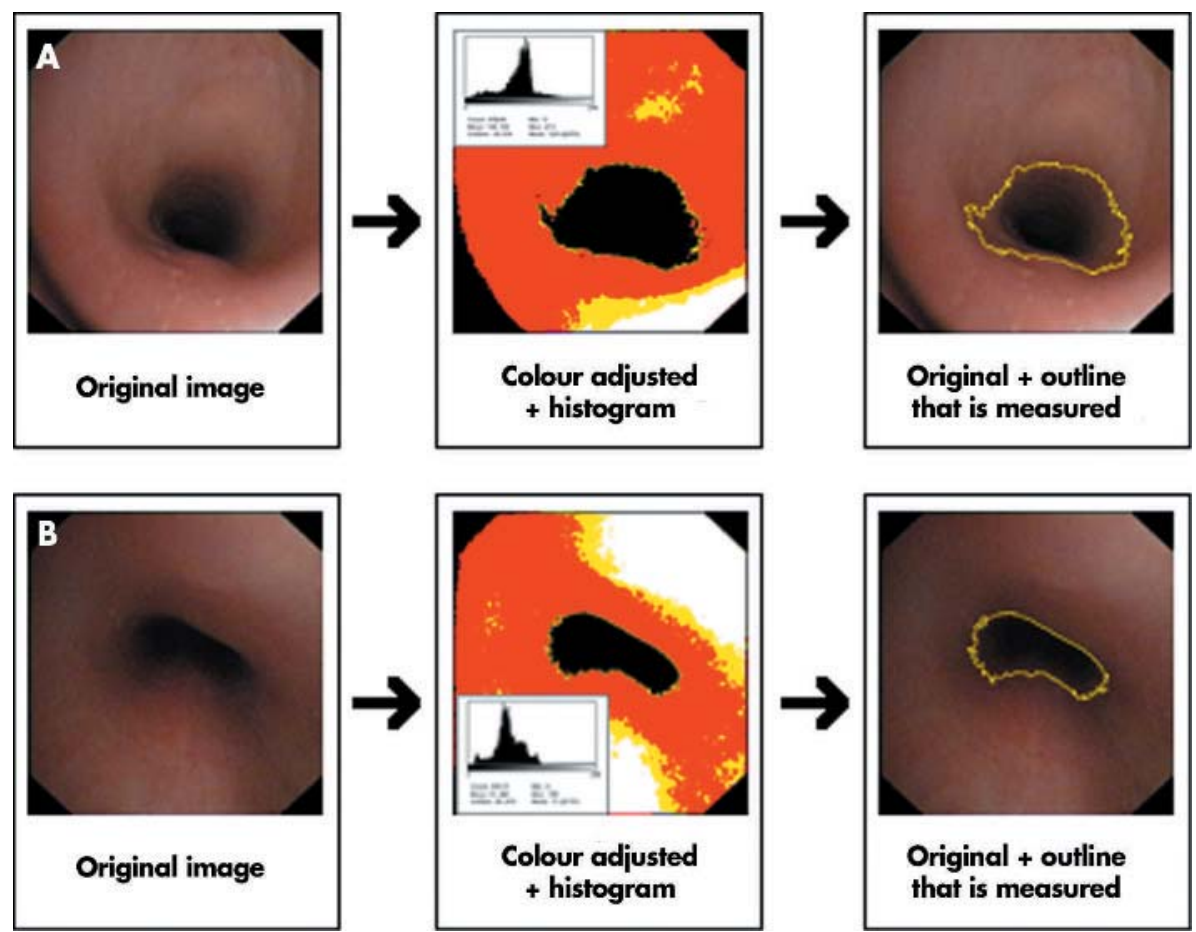

Figure 3A,B This series displays the cricoid measurement areas. (B) An area of tracheomalacia with anterior flattening from left to right and the pars bulging anteriorly. Note the slight overestimate of the cricoid size with the inclusion of the posterolateral angles. 
wedged position. Despite the relatively large lumina of the model, there was no "slack effect" - that is, the bronchoscope did not alter its position and it accurately retraced to its original zero point position. Images were then captured using the colour histogram mode technique. The radii of the validation tubes were calculated from the areas measured by the CHMT as described above, and compared with the real radii of the engineered tubes. The radii were calculated from the magnified area in accordance with the following formula: $\pi r_{\text {mag }}^{2}$ where $r_{\text {mag }}=$ radius real $\times$ magnification factor. Radii were also calculated from perimeter measurements and direct linear measurement of diameter via functions of the Image $J$ program.

\section{Reliability testing}

Digital video images obtained from a series of children who had undergone FVB (Olympus BF Type 3C160, Olympus, Tokyo, Japan) for chronic cough were opportunistically selected for assessment that included measurement of airways cross sectional area. Twenty eight end expiratory images from 18 patients of median age 30 months (range 2127 months) were used for analysis. All FVB procedures were performed under spontaneously breathing general anaesthesia using sevoflurane and oxygen. ${ }^{3523}$ Airway lumina were identified and measured at the following sites: cricoid cartilage ring $(n=4)$, left main stem bronchus $(n=4)$, right main stem bronchus $(n=7)$, left upper lobe bronchus $(n=3)$, left lower lobe bronchus $(n=4)$, bronchus intermedius $(n=2)$, and trachea at the site of tracheomalacia $(n=4)$. All images were taken when the tip of the bronchoscope was positioned $10 \mathrm{~mm}$ from the object (airway of interest). This was done by positioning the image on the FVB television monitor as close as possible to the centre of the field of view. The "zero point" was then defined at end expiration by touching the object (nearest carina to lumen) and placing a marking peg on the bronchoscope at the site of exit of the bronchoscope from the anaesthetic mask. In the malacia cases the most proximal or "downstream" end of the malacia segment was defined as the site of assessment and "zero point". The bronchoscope was then withdrawn $10 \mathrm{~mm}$ and advanced again to check that the zero point was correct. When this was achieved the bronchoscope was held in that position with a $10 \mathrm{~mm}$ wedge between the peg and the mask port.

\section{In vivo measurements}

The in vivo tests of the above technique were carried out by assessing the success rates of the technique on images taken from all FVBs on a further 35 children. This was a different group of children from those whose images had been used in the reliability testing and had undergone bronchoscopy as part of their investigation for protracted or chronic cough (more than 3 months) and/or associated wheeze. At each image site (cricoid, right main stem bronchus, left main stem bronchus, bronchus intermedius, left upper lobe bronchus, left lower lobe bronchus, and areas demonstrating tracheomalacia and bronchomalacia) 3-5 images were collected. End expiratory images at each site were collected within a 20 second epoch. In total, this took less than 5 minutes to collect all of the images. An "acceptable result" was defined as that which could be completed with the CHMT and was visually representative of the luminal outline as shown in the figs 1-3. Image group sites were considered acceptable if two of three images defined as acceptable were within $10 \%$ of the cross sectional area of each other. To assess the effects of light intensity on shadowing, in vivo images were captured at two different light settings for the same individual-normal and low operating light level, the latter being performed by simply reducing the light intensity meter by two or three notches
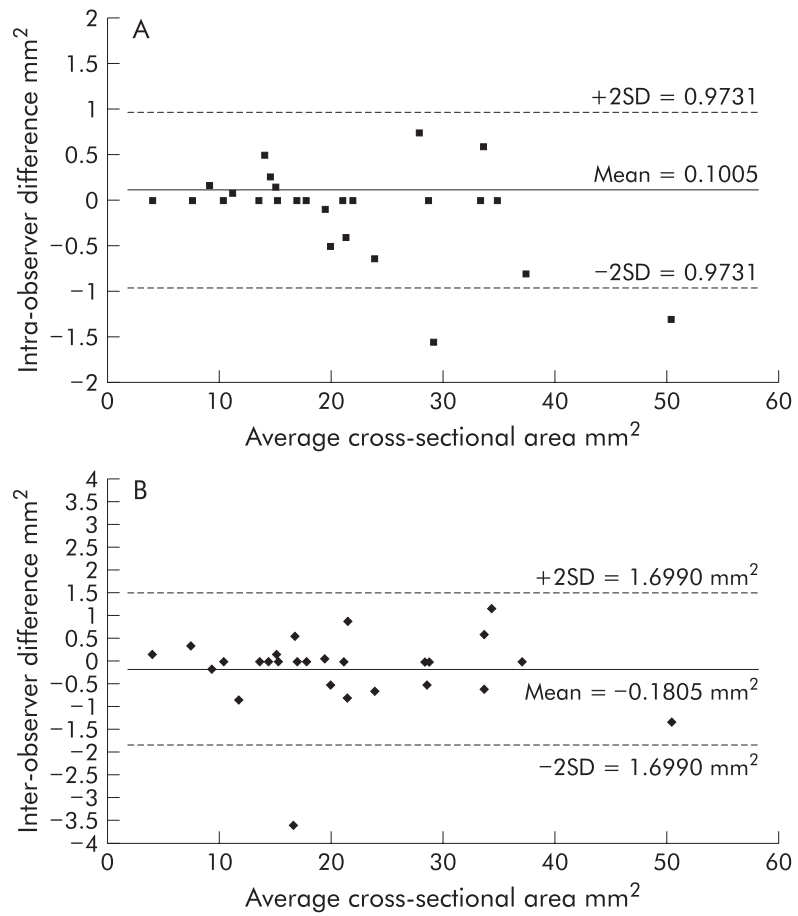

Figure 4 (A) Intra-observer and (B) inter-observer reliability assessments: Bland-Altman plots.

such that the visual contrast was optimally enhanced between the lumen and bronchial wall tissues. These in vivo images were compared with the measured visual assessment at these light settings. The visual assessment or the visual assessment modification of the CHMT used the same methodology with the colour histogram except the mode point was not used as the defining point of the binary assessment; instead, the histogram minimum and maximum brightness intensity line was moved so that as much as possible of the desired site of assessment was incorporated into the area being outlined and measured. This was done because it was observed that the normal light intensity tended to underestimate the areas of measurement at some sites within the airway while reduced light intensity appeared to approximate the real area perceived by visual inspection.

To estimate the applicability of these measurements to the clinical situation, comparisons of these areas were also made with external diameter derived cross sectional areas of endotracheal tube sizes that could be expected for the age of the child using the commonly used formula for endotracheal tube size: $(\mathrm{Age} / 4)+4{ }^{25}$ This was carried out on further children (total $n=116$ ) subsequently accrued after the initial pilot data had been gained from the first 35 patients.

The study was approved by the institutions ethics committee and written and informed consent was obtained from each child's parent.

\section{Statistical analysis}

Bland-Altman plots were used to examine intra-observer and inter-observer reliability expressed as repeatability coefficients $(1.96 \times \mathrm{SD})$. The Bland-Altman approach compares the difference between observations on the same subject using different measurement methods, and recognises that methods agree if the differences are small. Reliability ratings of measurements for continuous data from normal light, low light, and visual assessments of area were assessed using intraclass correlation expressed as an average reliability 


\begin{tabular}{|c|c|c|c|}
\hline & $\begin{array}{l}\text { Cricoid median area } \\
(\mathrm{sq} \mathrm{cm})\end{array}$ & $\begin{array}{l}\text { RMS median area } \\
(\mathrm{sq} \mathrm{cm})\end{array}$ & $\begin{array}{l}\text { LMS median area } \\
(\mathrm{sq} \mathrm{cm})\end{array}$ \\
\hline $\mathrm{N}$ & 14 & 9 & 5 \\
\hline Low light CHMT & 0.3599 & 0.26454 & 0.21401 \\
\hline Visual method & 0.32069 & 0.24087 & 0.20029 \\
\hline Wilcoxon $p$ value & 0.048 & 0.499 & 0.715 \\
\hline $\mathrm{N}$ & 14 & 8 & 6 \\
\hline Normal light CHMT & 0.22411 & 0.18141 & 0.10732 \\
\hline Visual method & 0.25911 & 0.23048 & 0.20357 \\
\hline Wilcoxon $p$ value & 0.021 & 0.018 & 0.028 \\
\hline
\end{tabular}

coefficient. ${ }^{26}$ Success rates for satisfactory tests defined as "acceptable results" and coefficient of variation of repeated measurements of cross sectional area from the plastic model were expressed as percentages. Paired comparisons were made with a Wilcoxon pairs test with significance levels of $\leqslant 0.05$. All statistical analyses were carried out using SPSS for MAC version 11 (SPSS Inc, Chicago, IL, USA).

\section{RESULTS}

\section{Validation}

The validation results showed very high levels of agreement of measurements at all distances $(10 \mathrm{~mm}, 15 \mathrm{~mm}, 20 \mathrm{~mm}$ and $30 \mathrm{~mm}$ ) with the lowest variations at $30 \mathrm{~mm}$ and the highest at $15 \mathrm{~mm}$. The mean calculated radius from the measured areas was $0.94 \mathrm{~mm}$ and the coefficient of variation for this measurement was $7.5 \%$. Radii calculated from perimeter and direct diameter measurements were $1.06 \mathrm{~mm}$ and $1.05 \mathrm{~mm}$ and the coefficients of variation were $8.2 \%$ and $8.5 \%$, respectively.

\section{Reliability}

The intra-observer and inter-observer reliability of measurements performed on 28 images from 18 patients are shown in the Bland-Altman plots in fig 4 . The intra-observer repeatability coefficient was $0.9 \mathrm{~mm}^{2}$ while the inter-observer repeatability coefficient was $1.6 \mathrm{~mm}^{2}$ for images that had an average size of $20 \mathrm{~mm}^{2}$. This represents a $5-10 \%$ range in repeatability for these measurements. In practical terms, these are remarkably small differences and would be of little clinical concern.

\section{In vivo measurements}

The paired comparison of airway cross sectional areas from the cricoid, right main stem and left main stem bronchi using low light, normal light, and visual assessments is shown in table 1. While there were significant differences in cross sectional areas measured at all sites by the normal light CHMT and normal light visual assessment method, there were only significant differences between the low light CHMT and low light visual assessments at the cricoid. This was due to the tendency of the low light technique to incorporate the posterolateral angles of the cricoid into the measurements (see fig 3A). Normal light visual and low light visual assessments negated these additional posterolateral areas, and these assessments were not significantly different for all site comparisons ( $p=0.12$, Wilcoxon) or for the cricoid specific comparisons $(\mathrm{p}=0.33$, Wilcoxon $)$. The average intraclass correlation alpha value for normal light CHMT compared with normal light visual assessment was 0.90 (95\% CI 0.80 to 0.95 ), and for low light CHMT compared with low light visual assessment the average intraclass correlation alpha value was 0.93 (95\% CI 0.86 to 0.97 ).

The in vivo success rate of CHMT for an "acceptable result" was $91.7 \%$, with 673 of 734 images taken from 35 patients being completed by CHMT alone while the remaining $8.3 \%$ were completed by the visual assessment modification of CHMT. The success rate for the collection of two of three images whose cross sectional area measurements were within $10 \%$ of each other was $100 \%$. Comparisons of the airway lumen (cricoid) with the existing areas derived from the external diameters of the endotracheal tubes expected for the age of the patient are shown in table 2. There appears to be a tendency to underestimate in older children, but there is a wide range of sizes.

\section{DISCUSSION}

This is the first study defining an objective method of identification and measurement of airway lumen calibre. We have shown that the CHMT method is highly reliable and versatile in its capacities to be used in both the extrathoracic

\begin{tabular}{|c|c|c|c|c|c|}
\hline $\begin{array}{l}\text { Age range } \\
\text { (years) }\end{array}$ & $\mathbf{N}$ & $\begin{array}{l}\text { Mean cricoid } \\
\text { area } \\
(\mathrm{sq} \mathrm{cm})\end{array}$ & $\begin{array}{l}\text { Mean minimum } \\
\text { ET area } \\
(\mathrm{sq} \mathrm{cm})^{*}\end{array}$ & $\begin{array}{l}\text { Mean maximum } \\
\text { ET area } \\
(\mathrm{sq} \mathrm{cm})^{*}\end{array}$ & $\begin{array}{l}\text { ETT size } \\
\text { range }\end{array}$ \\
\hline $0-2$ & 54 & $\begin{array}{l}0.2972 \\
\text { Range } \\
0.24-0.44\end{array}$ & 0.2445 & 0.3020 & $4-4.5$ \\
\hline$>2-\leqslant 4$ & 31 & $\begin{array}{l}0.3584 \\
\text { Range } \\
0.32-0.46\end{array}$ & 0.3020 & 0.3741 & $4.5-5.5$ \\
\hline$>4-\leqslant 12$ & 31 & $\begin{array}{l}0.3832 \\
\text { Range } \\
0.23-0.54\end{array}$ & 0.3741 & 0.4538 & $5.5-7.5$ \\
\hline
\end{tabular}

*Calculated area is based on the external diameter of the age appropriate endotracheal tube (ETT). 
and intrathoracic large airways. The study has shown that the technique has very high intra-observer and inter-observer reliability and that the cross sectional area as assessed at "low light intensity" is more likely to be representative of the true cross sectional area than that captured at "normal operating light" across a variety of lesions and airway sizes. In the clinical situation this means that the most representative or visually true area for measurement can be obtained from simply racking the light intensity meter down two or three notches. This process does not change the capacity of the technique to identify the lumen objectively. In view of the simplicity of the technique and the "user friendly" inexpensive software, we speculate that this new method might be applicable to all forms of flexible videoscopic work. In addition, with further developments of its user independent attributes, this technique offers the potential of a computerbased real time measurement that can be incorporated into the routine workings of this type of bronchoscope.

The validation measurement variability with our technique was excellent with a coefficient of variation in area of $7.5 \%$. As the bronchoscope was "hand held", slight movement differences within the tip of the bronchoscope and therefore its orientation to the object were possible. The random scatter of the points that were greater than $5 \%$ of the real measure strongly supports this and implies that the cross sectional area measurements are also highly reliable and representative of real life situations. Currently, as shown in table 2, there does appear to be a tendency to underestimate the size of the cricoid in older patients. This is more likely to be a function of bronchoscopic positioning $10 \mathrm{~mm}$ from the object than a limitation of the measurement tool and process itself. ${ }^{24}$ However, while cricoid size-as defined by diameter measurements with MRI-appears to be linearly related to size, it does have a very wide range of ages for any one measurement distance. $^{15} 27$

Furthermore, our in vivo data are within acceptable levels of agreement with the existing (though limited) data on tracheal cross sectional areas from CT scanning, and even with the recent videoscope and ultrasound data involving cricoid measurements only. ${ }^{15}{ }^{28-33}$ This level of agreement has to be considered in the context of different anatomical sites, a number of methodological differences, and the fact that many CT studies have shown extensive intra-subject variations in tracheal measurements across sites and the respiratory cycle. ${ }^{28}{ }^{29}$ 32-34 The methodological differences between our study and CT studies relate to the effects of anaesthesia, lung volume, respiratory timing, and averaging calculation methods. For example, Griscom sampled infants at near functional residual capacity and older children with a breath hold at or near total lung capacity, and there were a variety of states of consciousness (awake and/or sedated) within the group. ${ }^{28-31}$ The assessment site and conditions of our patients were much more uniform in that we selected the cricoid at end expiration in anaesthetised patients spontaneously breathing with oxygen and sevoflurane. Sevoflurane has a dose dependent bronchodilating effect on bronchoconstricted proximal airways but it is not known to cause bronchodilatation in unconstricted airways. ${ }^{35}$ We have previously shown that this anaesthetic and bronchoscopic technique may be associated with degrees of hypercapnia, but this effect is more likely to be related to minute ventilation effects than to isolated lung volume effects. ${ }^{5}$ As the cricoid is a complete ring, it is highly unlikely that any of these or associated factors would have altered the measurements of area in any inconsistent way, thus enhancing the robustness of our results. The trachea is known to undergo dynamic changes across the respiratory cycle, whereas the cricoid (being a complete ring) is not subject to such variations. ${ }^{29}$ In our study we assessed cricoid size at the end of expiration because, at this point in time, the cross sectional area of the extrathoracic airway is maximal but, more importantly, the dynamic action of the trachea during inspiration is to lengthen, thus moving the object of interest in the field of view away from the lens and consequently altering the magnification factors. CT studies have generally not only discounted the effects of the respiratory cycle, but also the point of measurement can be difficult to ascertain radiologically and they have therefore used averaging over a number of sites. The zero point of our measurements was at end expiration for the aforementioned reasons, and also because the end expiratory pause period was readily definable and therefore a period where measurement was logistically easy.

While it might be argued that a real time comparison of our measurement with that of a CT scan should have been made, there were and are a number of limitations to this means of dual assessment in the paediatric population which negated this approach. Firstly, radiation scatter of the metallic tip of the bronchoscope is likely to disrupt the capacity for accurate reconstruction and measurement of the sites concerned. In addition, if the measurements are not done concomitantly, the radiation dose required for construction of a virtual CT scan would be increased and there could be no regulation or control of lung volumes to ensure an ideal comparison. Finally, there are ethical considerations of increased cancer risk associated with paediatric CT scanning, especially with repeated measurements that would be required for follow up. $^{36}$

The factors that govern tissue reflectance and absorption of light during the respiratory cycle while under anaesthesia are complex and, no doubt, are compounded by the physical effects of the instrument, the type of light, airway suctioning, and disease processes. ${ }^{37-39}$ The effects of severely desaturated tissue are unknown for this method, but the oxygen saturation level of haemoglobin is only one of many factors that govern reflectance from tissues. While these issues need to be elucidated, this technique is very simple and could be easily applied to any clinical bronchoscopic service as it does allow for accurate representation of the lumen despite all of the above factors being present in many of the patients assessed. Our CHMT is similar to that described by Kumar et al. ${ }^{6}$ They used an 8-bit histogram binary technique to define the gut lumen in which grey level thresholding was used to find the darker areas of an endoscopic image. However, when we applied a similar black and white (binary) technique to our airway images, it did not identify the lumen with adequate success rates.

A real time and automated measurement system that allowed immediate access to airway cross sectional area and percentage predicted assessments for age or other indices of growth would be advantageous to a bronchoscopist. This is not currently available, but this technique of automated identification and measurement of lumen brings us a step nearer. In conclusion, CHMT applied to FVB images provides a versatile, representative, and accurate method of measuring airway dimensions. This technique therefore has enormous potential for documenting serial changes in a whole range of congenital and acquired airway lesions encountered in paediatric bronchoscopy. The objective nature of the measurements also provides the basis for a real time and automated measurement technique to be developed and applied to routine bronchoscopic work. It may also lead to a tool for three dimensional (3D) reconstruction of the paediatric airways and airway lesions.

\section{ACKNOWLEDGEMENTS}

The authors acknowledge the interest and support of Dr S Wilson and Dr A Zvyagin from the Departments of ITEE and Physics at the 
University of Queensland. The authors also thank the physicians, in particular Dr J Marchant and Dr N Dore, for their clinical support, and Dr J Wuth and Dr L Patterson for their anaesthetic expertise and care during the bronchoscopic procedures.

\section{Authors' affiliations}

I B Masters, P W Francis, A B Chang, Department of Respiratory Medicine, Royal Children's Hospital, Herston 4029, Brisbane, Australia I B Masters, P W Francis, A B Chang, School of Medicine, Discipline of Paediatric and Child Health, University of Queensland, Herston 4029, Brisbane, Australia

M M Eastburn, University of Queensland, Department of Information Technology and Electrical Engineering, St Lucia 4072, Brisbane, Australia

R Wootton, University of Queensland Centre for Online Health, Royal Children's Hospital, Herston 4029, Brisbane, Australia

R S Ware, School of Population Health, The University of Queensland, Herston 4006, Brisbane, Australia

P V Zimmerman, Department of Thoracic Medicine, The Prince Charles Hospital, Chermside 4032, Brisbane, Australia

\section{REFERENCES}

1 Wood R, Prakash U. Pediatric flexible bronchoscopy. In: Prakash UBS, ed. Bronchoscopy. New York: Raven Press, 1994:345-56.

2 Nicolai T. Pediatric bronchoscopy. Pediatr Pulmonol 2001;31:150-64.

3 Masters IB, Cooper P. Paediatric flexible bronchoscopy. J Paediatr Child Health 2002;38:555-9.

4 Midulla F, de Blic J, Barbato A, et al. Flexible endoscopy of paediatric airways. Eur Respir J 2003;22:698-708

5 Chang AB, Moloney GE, Harms PJ, et al. Endoscopic intratracheal carbon dioxide measurements during pediatric flexible bronchoscopy. Paediatr Anaesth 2004; 14:650-5.

6 Kumar S, Asari KV, Radhakrishnan D. Real-time automatic extraction of lumen region and boundary from endoscopic images. Med Biol Eng Comput 1999:37:600-4.

7 Panitch HB, Talmaciu I, Heckman J, et al. Quantitative bronchoscopic assessment of airway collapsibility in newborn lamb tracheae. Pediatr Res 1998;43:832-9.

8 Rozycki HJ, Van Houten ML, Elliott GR. Quantitative assessment of intrathoracic airway collapse in infants and children with tracheobronchomalacia. Pediatr Pulmonol 1996;21:241-5.

9 Vakil N. Measurement of lesions by endoscopy:an overview. Endoscopy 1995;27:694-7

10 Vakil N, Bourgeois K. A prospective, controlled trial of eight-bit, 16-bit, and 24-bit digital color images in electronic endoscopy. Endoscopy 1995;27:589-92.

11 Vakil N, Smith W, Bourgeois K, et al. Endoscopic measurement of lesion size: improved accuracy with image processing. Gastrointest Endosc 1994:40:178-83.

12 Riff EJ, Mitra S, Baker MC. Pediatric fiberoptic video bronchoscopy: the use of computer interfacing. Comput Biol Med 1993;23:345-7.

13 Dorffel WV, Fietze I, Hentschel D, et al. A new bronchoscopic method to measure airway size. Eur Respir J 1999;14:783-8.

14 Forkert L, Watanabe H, Sutherland K, et al. Quantitative videobronchoscopy: a new technique to assess airway caliber. Am J Respir Crit Care Med 1996;154:1794-803

15 Husein M, Manoukian JJ, Platt R, et al. Ultrasonography and videobronchoscopy to assess the subglottic diameter in the paediatric population: a first look. J Otolaryngol 2002;31:220-6.
16 Ray S. Applied photographic optics. London: Focal Press (Butterworth Group), 1988.

17 McFawn PK, Forkert L, Fisher JT. A new method to perform quantitative measurement of bronchoscopic images. Eur Respir J 2001;18:817-26.

18 Cotton R. Pediatric laryngotracheal stenosis. J Pediatr Surg 1984; 19:699-704

19 Jesseph JM, Merendino KA. The dimensional interrelationships of the components of the human tracheobronchial tree. Surg Gynecol Obstet 1957;105:210-4.

20 Weibel E. Airways and blood vessels. In: The pathway for oxygen structure and function in the mammalian respiratory system. Cambridge: Harvard University Press, 1984:272-301.

21 Weibel E. Morphometrics of the lung. In: Fenn W, ed. Handbook of physiology section 3: Respiration. Volume 1. Baltimore, MD: Williams and Wilkins, 1964:285-307.

22 Brodsky J, Malott K, Angst M, et al. The relationship between tracheal width and left bronchial width: implications for left-sided double lumen tube selection. J Cardiothorac Vasc Anesth 2001;15:216-7.

23 Masters IB, Chang AB, Patterson L, et al. Series of laryngomalacia, tracheomalacia, and bronchomalacia disorders and their associations with other conditions in children. Pediatr Pulmonol 2002;34:189-95.

24 Masters IB, Eastburn M, Francis PW, et al. Quantification of the magnification and distortion effects of a pediatric flexible video-bronchoscope. Respir Res 2005;6:16.

25 Shann F. Drug doses, 12th ed. Melbourne: Child Health Information Centre, $\mathrm{RCH}, 2003$.

26 Armitage P, Berry G, Matthews J. Statistical methods in medical research, 4th ed. Massachusetts: Blackwell Publishing Company, 2002.

27 Litman RS, Weissend EE, Shibata D, et al. Developmental changes of laryngeal dimensions in unparalyzed, sedated children. Anesthesiology 2003:98:41-5.

28 Griscom NT. Computed tomographic determination of tracheal dimensions in children and adolescents. Radiology 1982;145:361-4.

29 Griscom NT, Wohl ME. Tracheal size and shape: effects of change in intraluminal pressure. Radiology 1983;149:27-30.

30 Griscom NT, Wohl ME. Dimensions of the growing trachea related to body height. Length, anteroposterior and transverse diameters, cross-sectional area, and volume in subjects younger than 20 years of age. Am Rev Respir Dis 1985;131:840-4.

31 Griscom NT, Wohl ME. Dimensions of the growing trachea related to age and gender. AJR Am J Roentgenol 1986;146:233-7.

32 Effmann EL, Fram EK, Vock P, et al. Tracheal cross-sectional area in children: CT determination. Radiology 1983;149:137-40.

33 Newth CJ, Lipton MJ, Gould RG, et al. Varying tracheal cross-sectional area during respiration in infants and children with suspected upper airway obstruction by computed cinetomography scanning. Pediatr Pulmonol 1990;9:224-32.

34 Stern EJ, Graham CM, Webb WR, et al. Normal trachea during forced expiration: dynamic CT measurements. Radiology 1993;187:27-31.

35 Hashimoto $\mathrm{Y}$, Hirota $\mathrm{K}$, Ohtomo $\mathrm{N}$, et al. In-vivo direct measurement of the bronchodilating effect of sevoflurane using a superfine fiberoptic bronchoscope: comparison with enflurane and halothane. J Cardiothorac Vasc Anesth 1996;10:213-6.

36 Brenner DJ. Estimating cancer risks from pediatric CT: going from the qualitative to the quantitative. Pediatr Radiol 2002;32:228-3.

37 Tuchin V. Handbook of optical and biomedical diagnostics. Washington: SPIE-The International Society for Optical Engineering, 2002.

38 Andersen PE, Thrane L, Yura HT, et al. Advanced modelling of optical coherence tomography systems. Phys Med Biol 2004;49:1307-27.

39 Nilsson L, Johansson A, Kalman S. Macrocirculation is not the sole determinant of respiratory induced variations in the reflection mode photoplethysmographic signal. Physiol Meas 2003;24:925-37. 